\title{
Unusual injury pattern (cauterization)
}

\author{
Mohammed Madadin $^{1 *}$ and Khamis Almazrooei ${ }^{2}$
}

\begin{abstract}
Background: Pattern of scarring is unusual and the most likely explanation is cauterization (burn). Cauterization is a traditional method of treatment which has been used in some cultures for centuries. It is still a form of treatment in some parts of the world such as rural India, and the Middle East.

Case presentation: This is a case of a young male in his 30s. He was found dead in his bed in the early morning. On external examination, there were no significant findings other than the unusual pattern of old injury (scarring).

Conclusion: Forensic practitioner should consider the probability of cauterization-related scarring especially if the deceased has lived in areas where this practice is common.
\end{abstract}

Keywords: Scar, Burn, Cauterization, Bizarre injury, Unusual injury

\section{Background}

This pattern of scarring is unusual and the most likely explanation is cauterization (burn). Cauterization is a traditional method of treatment which has been used in some cultures for centuries. It is still a form of treatment in some parts of the world such as rural India, and the Middle East. It involves placing red-hot pieces of iron on the skin in what are believed to be "disease-specific" locations on the body. It has been used to treat both physical and mental disorders. In other settings, cautery is used directly on open wounds to stop bleeding, sterilize wounds, drain abscesses, or even to remove skin tumors (Abou-Elhamd 2009; Mohammad 2015).

Cauterization injuries are thermal injuries leaving scar(s) that usually takes the shape of the equipment used. It often has well-defined edges and usually involves specific sites on the body. Many patients will have multiple cauterization scars with symmetrical distribution, a feature which can help to identify the therapeutic nature of their infliction. The history is important in such correlation (Mohammad 2015).

\section{Case presentation}

This is a case of a young male in his 30s who came to Australia as a refugee from Afghanistan. He was found dead in his bed in the early morning. The deceased had no past medical history. He was not a smoker or a known drug user.

On external examination, there were no significant findings other than the following pattern of old injury.

On the umbilicus, there was a healed scar, oval in shape, measuring $2 \times 3 \mathrm{~cm}$, obliterating the umbilicus. On the back, at the same level of the wound, there was another similarly shaped healed scar of the same size (Figs. 1, 2, 3, and 4). The internal examination showed significant coronary atherosclerosis and ischemic heart disease. There was no significant injury or pathology in the abdomen or at the scar sites.

\footnotetext{
* Correspondence: Mmadadin@iau.edu.sa

'Department of Pathology, University of Dammam, Dammam, Saudi

Arabia

Full list of author information is available at the end of the article
} 


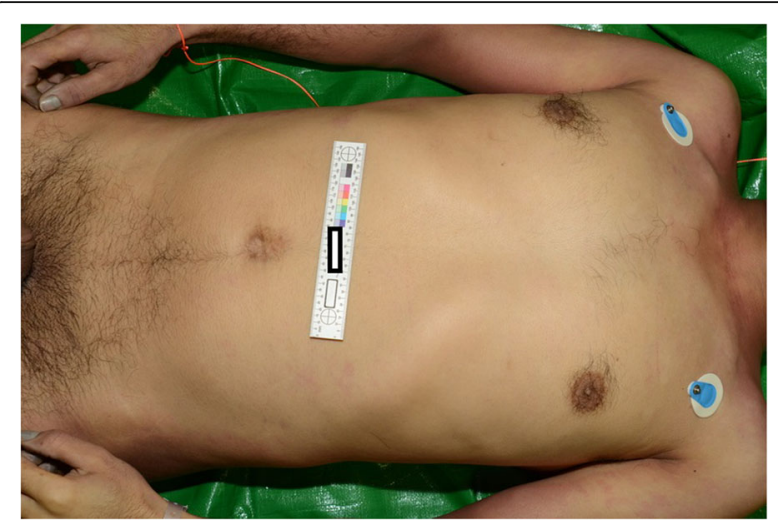

Fig. 1 View of the umbilical scar and anterior body part

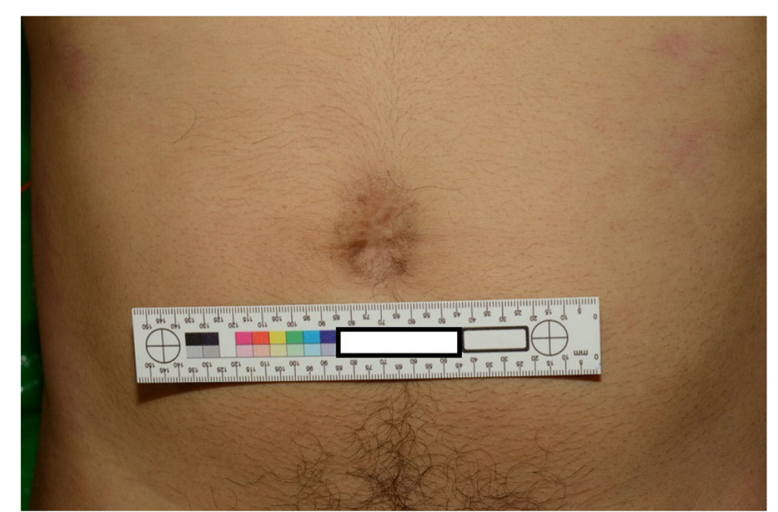

Fig. 2 Close view of the umbilical scar

\section{Conclusion}

Forensic practitioner should consider the probability of cauterization-related scarring especially if the deceased has lived in areas where this practice is common.

\section{Acknowledgments}

We would acknowledge the Victorian Institute of Forensic Medicine where the death was investigated.

\section{Funding}

No funding was granted.

\section{Availability of data and materials}

Data and materials are available.

\section{Authors' contributions}

Both authors examined the case, wrote and reviewed the manuscript. All authors read and approved the final manuscript.

\section{Ethics approval and consent to participate}

Approval of publication was obtained from Victorian Institute of Forensic Medicine.

\section{Consent for publication}

Manuscript does not include any personal data. Consent from the deceased family was not obtained.

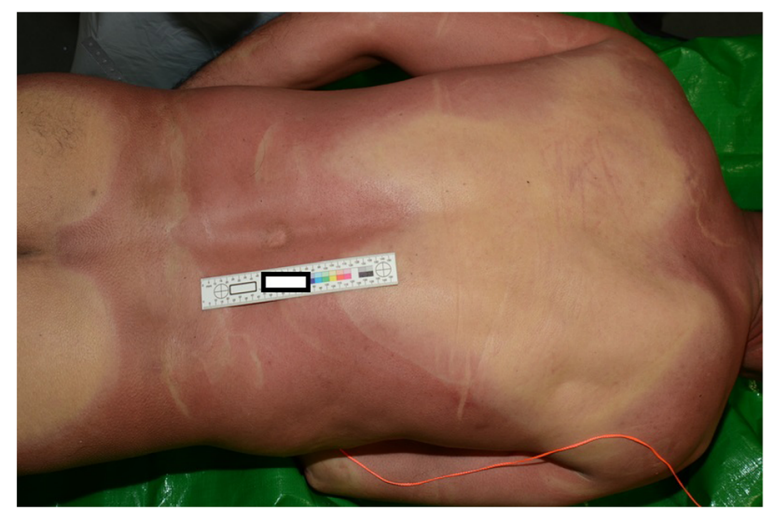

Fig. 3 View of the back and the scar on the back

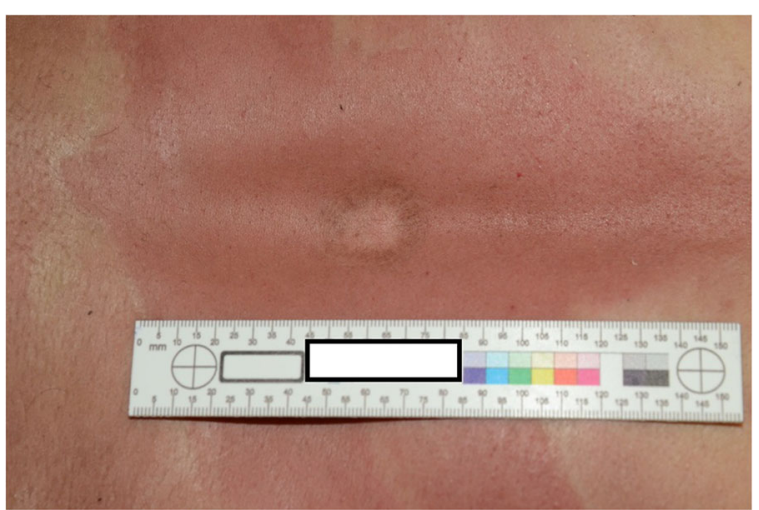

Fig. 4 Close view of the back scar

\section{Competing interests}

The authors declare that they have no competing interest.

\section{Publisher's Note}

Springer Nature remains neutral with regard to jurisdictional claims in published maps and institutional affiliations.

\section{Author details}

'Department of Pathology, University of Dammam, Dammam, Saudi Arabia. ${ }^{2}$ Victorian Institute of Forensic Medicine, Melbourne, Victoria, Australia.

Received: 1 December 2018 Accepted: 27 December 2018 Published online: 07 January 2019

\section{References}

Abou-Elhamd KEA (2009) Kaiy as traditional therapy for pain: is it helpful or a myth? The Journal of Laryngology \& Otology 123:566-568

Mohammad Y, Al-Ahmari A, Al-Dashash F, Al-Hussain F, Al-Masnour F et al (2015) Pattern of traditional medicine use by adult Saudi patients with neurological disorders. BMC Complement Altern Med 15:102 